\title{
REMARK ON THE PURITY OF THE BRANCH LOCUS
}

\section{ERNST KUNZ}

The purpose of the following note is to give a simple proof for the purity of the branch locus for local rings of characteristic $p>0$.

Let $R$ be a regular local ring of characteristic $p>0, K$ its quotient field, $k$ its residue class field. Let $L$ be a finite separable extension of $K$ and $S$ be the integral closure of $R$ in $L$. It is well known that $S$ is a finitely generated $R$-module and that $S=\bigcap_{h(\mathfrak{B})=1} S_{\mathfrak{P}}$, where $\mathfrak{P}$ runs over all prime ideals of $S$ of height $h(\mathfrak{B})=1$.

Lemma 1. $R$ is a faithfully flat $R^{p}$-module.

This follows from the commutative diagram

$$
\begin{aligned}
& R \rightarrow \hat{R}=k\left[\left[x_{1}, \cdots, x_{n}\right]\right] \\
& \uparrow \\
& R^{p} \rightarrow \hat{R}^{p}=k^{p}\left[\left[x_{1}^{p}, \cdots, x_{n}^{p}\right]\right],
\end{aligned}
$$

where $\hat{R}$ is the completion of $R, \hat{R}=k\left[\left[x_{1}, \cdots x_{n}\right]\right]$, a representation of $\hat{R}$ as a ring of formal power series over $k$, and from the fact that the extensions $R \rightarrow \hat{R}, R^{p} \rightarrow \hat{R}^{p}$ and $k^{p}\left[\left[x_{1}^{p}, \cdots x_{n}^{p}\right]\right] \rightarrow k\left[\left[x_{1}, \cdots x_{n}\right]\right]$ are faithfully flat.

REMARK. If $R$ is a commutative ring, $p$ an integer, $T$ a multiplicatively closed subset of $R$, then $R_{T}=R_{T^{p}}$.

Lemma 2. $R\left[S^{p}\right] \cong R \otimes_{R^{p}} S^{p}$.

Consider the sequence

$$
R \otimes_{R^{p}} S^{p} \rightarrow R \otimes_{R^{p}} L^{p} \rightarrow K \otimes_{K^{p}} L^{p} \rightarrow K\left[L^{p}\right] \subseteq L .
$$

The first arrow is an injection by Lemma 1 , the second is an isomorphism, as $R \otimes_{R^{p}} L^{p} \cong\left(R \otimes_{R^{p}} K^{p}\right) \otimes_{K^{p}} L^{p}$ and $K \cong R \otimes_{R^{p}} K^{p}$ by the remark. The third arrow is an isomorphism, as $L$ is separable over $K$. As $R\left[S^{p}\right]$ is the image of $R \otimes_{R^{p}} S^{p}$ in $K\left[L^{p}\right]$, the lemma follows.

Lemma 3. $R\left[S^{p}\right]=\bigcap_{h(q)=1} R\left[S^{p}\right]_{q}$, where $q$ runs over all prime ideals of $R\left[S^{p}\right]$ of height 1 .

For each $\mathfrak{q}$ there is exactly one prime ideal $\mathfrak{P}$ of $S$ with $\mathfrak{P} \cap R\left[S^{p}\right]$ $=\mathfrak{q}$, namely

$$
\mathfrak{B}=\sqrt{ } \mathfrak{q}=\left\{x \in S \mid x^{p} \in \mathfrak{q}\right\},
$$

Received by the editors September 21, 1967. 
and $h(\mathfrak{B})=h(\mathfrak{q})$. By the remark and by Lemma 2 we have $R\left[S^{p}\right]_{a}$ $\cong R \otimes_{R^{p}}\left(S_{\mathfrak{B}}\right)^{p}$. Let

$$
y \in \bigcap_{h(\mathfrak{B})=1} R \otimes_{R^{p}}\left(S_{\mathfrak{B}}\right)^{p} \subseteq R \otimes_{R^{p}} L^{p}
$$

Then

$$
y=\sum_{i=1}^{m} r_{i} \otimes l_{i} \quad\left(r_{i} \in R, l_{i} \in L^{p}\right) .
$$

Since $S^{p}$ is an integrally closed noetherian domain, the $l_{i}(i=1 \cdots m)$ are contained in almost all of the $\left(S_{\mathfrak{B}}\right)^{p}$. Let $\mathfrak{B}_{1}, \cdots, \mathfrak{B}_{\text {r }}$ be the exceptional prime ideals. Then

$$
y \in R \otimes_{R^{p}}\left(\bigcap_{\mathfrak{B} \neq \mathfrak{B}_{i}}\left(S_{\mathfrak{B}}\right)^{p}\right) \cap \bigcap_{i=1}^{r} R \otimes_{R^{p}}\left(S_{\mathfrak{B}_{i}}\right)^{p} .
$$

Since $R$ is flat over $R^{p}$ the tensor product commutes with finite intersections (Bourbaki, Eléments demathématique. Algèbre commutative, Hermann, Paris, Chapter I, §2, Proposition 6). Thus

$$
y \in R \otimes_{R^{p}}\left(\bigcap_{h(\mathfrak{B})=1}\left(S_{\mathfrak{B}}\right)^{p}\right)=R \otimes_{R^{p}} S^{p} .
$$

Lemмa 4. Let $\mathfrak{B} \in \operatorname{Spec}(S), \mathfrak{B} \cap R\left[S^{p}\right]=\mathfrak{q}$. $\mathrm{S}_{\mathfrak{P}}$ is unramified over $R$ if and only if $S_{\mathfrak{B}}=R\left[S^{p}\right]_{\mathrm{q}}$.

Let $\mathfrak{B} \cap R=\mathfrak{g}$ and $k_{\mathfrak{B}}, k_{\mathfrak{q}}, k_{\mathfrak{g}}$ be the residue class fields of $S_{\mathfrak{B}}$, $R\left[S^{p}\right]_{\mathfrak{q}}$ and $R_{\mathfrak{g}}$ respectively. Then $k_{\mathfrak{q}}=k_{\mathfrak{g}}\left[k_{\mathfrak{B}}^{p}\right]$.

If $S_{\mathfrak{B}}$ is unramified over $R$, then it is also unramified over $R\left[S^{p}\right]$. It follows that $k_{\mathfrak{B}}=k_{\mathfrak{q}}=k_{\mathfrak{g}}\left[k_{\mathfrak{B}}^{p}\right]$, as $k_{\mathfrak{B}}$ is at the same time separable and purely inseparable over $k_{\mathfrak{q}} . S_{\mathfrak{P}}$ is a finitely generated $R\left[S^{p}\right]_{q^{-}}$ module by the above remark and $S_{\mathfrak{F}}=R\left[S^{p}\right]_{\mathfrak{q}}+q S_{\mathfrak{F}}$. By the lemma of Nakayama, we have $S_{\Re}=R\left[S^{p}\right]_{q}$.

Conversely, let $S_{\mathfrak{P}}=R\left[S^{p}\right]_{\mathrm{q}}$. Then $k_{\mathfrak{B}}=k_{\mathfrak{q}}=k_{\mathfrak{g}}\left[k_{\mathfrak{B}}^{p}\right]$. Since $k_{\mathfrak{B}}$ is finite over $k_{\mathfrak{g}}$ it follows that $k_{\mathfrak{B}}$ is separable over $k_{\mathfrak{g}}$ and $k_{\mathfrak{B}} \cong k_{\mathfrak{g}} \otimes_{\left(k_{\mathfrak{g}}\right)^{p}}\left(k_{\mathfrak{P}}\right)^{p}$. The ideal $i=\mathfrak{g} R_{\mathfrak{g}} \otimes\left(S_{\mathfrak{B}}\right)^{p}+R_{\mathfrak{g}} \otimes\left(\mathfrak{B} S_{\mathfrak{B}}\right)^{p}$ of

$$
R_{\mathfrak{B}} \otimes_{\left(R_{\mathfrak{g}}\right)^{p}}\left(S_{\mathfrak{B}}\right)^{p} \cong R\left[S^{p}\right]_{\mathfrak{g}}=S_{\mathfrak{B}}
$$

is the maximal ideal of this ring, as $R_{\mathfrak{B}} \otimes_{\left(R_{\mathfrak{g}}\right.}{ }^{p}\left(S_{\mathfrak{B}}\right)^{p} / i \cong k_{\mathfrak{g}} \otimes_{\left(k_{\mathfrak{g}}\right)^{p}}\left(k_{\mathfrak{B}}\right)^{p}$ is a field, thus $\mathfrak{B} S_{\mathfrak{P}}=\mathfrak{g} S_{\mathfrak{B}}+\mathfrak{B}^{p} S_{\mathfrak{B}}$. From the lemma of Nakayama, we have $\mathfrak{B} S_{\mathfrak{P}}=\mathfrak{g} S_{\mathfrak{P}}$ and $S_{\mathfrak{P}}$ is unramified over $R$.

THEOREM. Let $S_{\mathfrak{B}}$ be unramified over $R$ for all prime ideals $\mathfrak{B}$ of $S$ 
with height 1. Then $S$ is unramified over $R .^{1}$

Let $\mathfrak{B} \in \operatorname{Spec}(S)$ be a prime ideal of height 1 and $q=\Re \cap R\left[S^{p}\right]$. Then by Lemma 4 we have $S_{\mathfrak{B}}=R\left[S^{p}\right]_{\mathfrak{q}}$. Lemma 3 gives us $S=\bigcap_{h(\mathcal{B})=1} S_{\mathfrak{B}}$ $=\bigcap_{h(q)=1} R\left[S^{p}\right]_{a}=R\left[S^{p}\right]$. Now by Lemma $4, S$ is unramified over $R$. It is easy to extend Lemma 4 to the following criterion: Let $R \subseteq S$ be noetherian local rings with maximal ideals $m \subseteq M$ and residue class fields $k \subseteq K$. If char $k=p>0$, if $K$ is finite over $k$ and $S$ is a finitely generated $R\left[S^{p}\right]$-module, then $S$ is unramified over $R$ if and only if $S=R\left[S^{p}\right]$.

University of Heidelberg, West Germany

1 ADDED IN PROOF. It is sufficient to assume that $R$ is a local domain, which is flat over $R^{p}$. However, in a forthcoming paper (Amer. J. Math.) the author will show that $R$ is then a regular local ring. 